\title{
Treat patient, not just the disease: holistic needs assessment for haematological cancer patients
}

\author{
Md Serajul Islam ${ }^{1,2}$ \\ ${ }^{1}$ Department of Haematology, Guy's \& St. Thomas Hospital, London; ${ }^{2}$ Department of Haematology, Broomfield \\ Hospital, Chelmsford, UK
}

\begin{abstract}
Haematological malignancies can have devastating effects on the patients' physical, emotional, psycho-sexual, educational and economic health. With the improvement of therapies patients with these malignancies are living longer, however significant proportion these patient show poor quality of life (QoL) due to various physical and psychological consequences of the disease and the treatments. Health-related QoL (HRQoL) is multi-dimensional and temporal, relating to a state of functional, physical, psychological and social/family well-being. Compared with the general population, HRQoL of these patients is worse in most dimensions. However without routine holistic need assessment (HNA), clinicians are unlikely to identify patients with clinically significant distress. Surviving cancer is a chronic life-altering condition with several factors negatively affecting their QoL, such as psychological problems, including depression and excessive fear of recurrence, as well as social aspects, such as unemployment and social isolation. These need to be adequately understood and addressed in the healthcare of long-term survivors of haematological cancer. Applying a holistic approach to patient care has many benefits and yet, only around $25 \%$ of cancer survivors in the UK receive a holistic needs assessment. The efforts of the last decade have established the importance of ensuring access to psychosocial services for haematological cancer survivors. We need to determine the most effective practices and how best to deliver them across diverse settings. Distress, like haematological cancer, is not
\end{abstract}

\footnotetext{
Correspondence: Md Serajul Islam, Department of haematology, Broomfield hospital, Court Road, Chelmsford, CM1 7ET, UK.

Tel.: +44.7769580452 - Fax: +44.1245.516669.

E-mail: serajul@doctors.org.uk
}

Key words: Haematological cancer; holistic needs assessment; lymphoma; myeloma; leukaemia.

Acknowledgments: the author acknowledges Talhah Saad Bin-Islam for his critical comment on the manuscript.

Conflict of interest: the author has no conflict of interest.

Received for publication: 11 May 2018.

Accepted for publication: 20 June 2018

This work is licensed under a Creative Commons Attribution NonCommercial 4.0 License (CC BY-NC 4.0)

CCopyright Md S. Islam1, 2018

Licensee PAGEPress, Italy

Oncology Reviews 2018; 12:374

doi:10.4081/oncol.2018.374 a single entity, and one treatment does not fit all. Psychosocialoncology needs to increase its research in comparative effectiveness.

\section{Introduction}

Haematological cancers are estimated to represent about $6.5 \%$ of all cancers worldwide in 2012. ${ }^{1}$ Haematological cancer is an Umbrella term which includes various forms and types of lymphomas (both Hodgkin's and non-Hodgkin's lymphoma), plasma cell malignancies, leukaemia (acute and chronic, lymphoid as well as myeloid) involving all body parts. These types of malignancies can affect any age groups from infants to extreme old group and can have devastating effect on a person, on a family and by enlarge can have enormous negative impact on the patients physical, emotional, psycho-sexual, educational and economic health.

Survival rates for haematological cancer continue to improve. The 5-year survival rates vary from $47 \%$ to $95 \%$ depending on the type of haematological malignancy. ${ }^{2}$ The number of cancer survivorsin the UK (currently 2 million) is projected to rise to 3 million by $2040 .{ }^{3}$ High levels of psychosocial distress have been found in patients with haematological cancer., ${ }^{4} 5$ In one recent study which included 718 patients showed that among the longterm haematological cancer survivors $15 \%$ reported significant psychological distress, $18 \%$ high levels of fatigue and $10 \%$ moderate to severe functional impairment. These groups of participants also showed poorer quality of life (QoL). ${ }^{6}$ However without routine holistic needs assessment (HNA), clinicians are unlikely to identify patients with clinically significant distress. ${ }^{7}$ In 2008 , the Institute of Medicine in USA found ample evidence to support a mandate that routine distress management be instituted across care sites. $^{8}$

In patients with hematologic malignancies symptoms related to the disease and its treatment remains a major problem in patient care. With the advent of new treatment including immunotherapy and targeted therapies the potential for prolonged life and additional treatment is increasing. Patient self-reported symptom burden during periods of observation and treatment are infrequently reported but is highly relevant to patient care. Health-related QoL (HRQoL) is multi-dimensional and temporal, relating to a state of functional, physical, psychological and social/family well-being. ${ }^{9}$ Compared with the general population, HRQoL of cancer patients is worse in most dimensions. ${ }^{10,11}$

Surviving cancer is a chronic life-altering condition with several factors negatively affecting their QoL, such as psychological problems, including depression ${ }^{12}$ and excessive fear of recurrence, as well as social aspects, such as unemployment ${ }^{13}$ and social isolation. ${ }^{14}$ These need to be adequately understood and addressed in the healthcare of long-term haematological cancer survivors. Assessment of patient-reported outcomes may allow for improvement in the management of symptoms and communication with 
providers. There is various symptom assessment tools are available that captures patient's perceived symptom burden that interferences with life issues in patients with hematologic malignancies. Early identification of patient symptom burden may allow for faster intervention and improvement in patient outcomes. In this article I have reviewed the currently available evidence forthe needs for holistic assessment of patients with haematological cancers.

\section{Search strategy}

A review was conducted from databases PubMed, Google scholar and Medline, searching for studies published between 1990 and 2017 with keywords: 'haematological cancer', 'quality of life', 'physical', 'psychological', 'social', 'vocational', 'professional', 'economic', 'cognitive', 'distress' and 'sexual' appearing in the abstracts. Various combinations were used to search for all databases i.e. 'QoL and haematological cancer, haematological cancer and physical, haematological cancer and psychological, haematological cancer and social, haematological cancer and cognitive, haematological cancer and economic, haematological cancer and professional, haematological cancer and vocational, haematological cancer and sexual, haematological cancer and distress. Search was repeated with words lymphoma, myeloma, leukaemia and haematological malignancy instead of haematological cancer.

\section{Inclusion criteria}

Prospective, comparative, exploratory, longitudinal or crosssectional studies, assessing the QoL or HRQoL were analysed. Papers focusing on lymphoma, leukaemia or myeloma patients with chemotherapy, chemo-immunotherapy, immunotherapy, radiotherapy or blood transfusion in periods of remission or relapse were included.

\section{Acute myeloid leukaemia and holistic needs assessment}

HRQoL has been recognized as a predictor of clinical progno$\operatorname{sis}^{15}$ and is a relevant consideration in disease management of AML. HRQoL assessments have become increasingly important in aiding in identifying and informing supportive therapy needs during treatment and beyond; however, HRQoL in leukaemia has received relatively minor attention. For individuals diagnosed with AML, intensive chemotherapy and bone marrow transplantation are still the first-line treatment options, often requiring long hospitalizations and severe treatment side effects; ${ }^{16}$ thereby significantly affecting their HRQoL. A review published in 2008 of HRQoL in leukemia randomized controlled trials (RCTs), including AML, highlighted the paucity of HRQoL data available in leukaemia research. ${ }^{17}$ Despite the importance of patient well-being, information on HRQoL of patients with AML and survivors of AML is lacking. ${ }^{16}$ Only limited number of studies have reported a global assessment of HRQoL and showed an improvement in HRQoL was generally shown among survivors compared to active AML. ${ }^{18-22}$

Leunis et al. reported anEuropean Quality of Life-5 Dimensions (EQ-5D) utility score of 0.82 , and an EQ visual analog scale (EQ-VAS) score of 74.6, a significantly lower result com- pared toa general population sample. ${ }^{23}$ On the contrary HRQoL in adult survivors of childhood AML showed no difference inphysical or mental scales compared to population norms suggesting childhood AML survivors do not seem to have long term deleterious consequences. ${ }^{24}$ Another study showed that patients actively undergoing treatment for AML, meanshort form (SF-36) physical scale scores were approximately two standard deviations below population norms within the first 6 weeks from diagnosis, indicating a quick and sharp decline in HRQoL shortly after diagnosis; mental scores were comparable with general norm scores. ${ }^{25} \mathrm{~A}$ comparison of mean scores between AML survivors and a general population sample found significantly lower scores reported across emotional, cognitive and social domains in the AML population. ${ }^{23}$

One prospective study showed except emotional functioning, there was a decline in various domains of HRQoL in the posttransplantation period, with the lowest scores at day 30 followed by subsequent slow improvement by day 365 . This pattern was similar for patients undergoing myeloablative conditioning (MAC) and reduced intensity conditioning (RIC). The RIC cohort had better quality of life at day 30 in the domains of physical functioning at day 30. A higher proportion of patients undergoing RIC had worsening of cognitive functioning from baseline from day 100 onward. ${ }^{26}$ A recent cross-sectional questionnaire study showed that patients who received chemotherapy alone had a better physical QOL than those who received allogeneic-haematopoietic stem cell transplant (Allo-HSCT). On the other hand, the allo-HSCT group reported a better mental QoL. Patients who had graft versus host disease (GvHD), experienced statistically and clinically significantly worse QoL than those who did not have GvHD. In the alloHSCT patients without GvHD, the physical QoL was comparable to that in the chemotherapy patients, and they experienced significantly better mental and general QoL than the chemotherapy patients. ${ }^{27}$

Another study comparing AML survivors to a general population sample identified a statistically significant negative impact on AML survivors in the fatigue, pain, dyspnoea and appetite loss domains. Active AML patients reported appetite loss and fatigue symptoms as having the most detrimental impact on HRQoL, whereas fatigue and insomnia had the worst impact on HRQoL among AML survivors. Perceived financial difficulties showed negative impact in both active AML patients and AML survivors and were reported to be significantly worse in AML survivors compared to ageneral population sample. HRQoL in relapsed participants was worse than in non-relapsed participants..$^{23}$ Thus it is quite clear that there is a definite need for routine holistic needs assessment for all leukaemia patients for better patient management, for better outcome and for better QoL.

\section{Myeloma and holistic needs assessment}

Multiple myeloma is an incurable cancer with a rising incidence globally. ${ }^{28}$ Myeloma is a haematological cancer affecting patients and their carers in many physical, emotional and social ways. Therefore, management should, if possible, be tailored to individual needs. The symptoms of myeloma and the side-effects of treatment may result in long-term disability and preclude many patients from returning to work. High-dose and conventional chemotherapy regimens also make employment impractical for periods of several months. Patients commonly need advice on socio-economic problems resulting from the condition and its treatment. The specialist team needs to be able to provide information on state benefits, e.g. disability living allowance and other appropriate social services. ${ }^{29}$ 
Less toxic treatments are increasingly available, so patients are living longer and treatment decisions are increasingly guided by QOL concerns. Despite these advances, from the initial diagnosis to the end of life, symptom control and preservation of QoL can be challenging. Myeloma causes destruction of the bones, bone marrow failure and renal failure, leading to impairments in physical, psychological and social domains of quality of life (QOL). ${ }^{30-32}$ Hence it has been recommended that QoL assessment should form part of the routine care of myeloma patients. ${ }^{30-33}$ Myeloma diagnosis and it is treatment causes various symptoms; however the presence or absence of physical symptoms per se was not the most important determinant of QoL, rather the impact of symptoms on other domains such as activities, participation, and emotional wellbeing was found to be the determinant of QoL. ${ }^{34}$

A key principle of the National Health Service (NHS)-based National Cancer Survivorship Initiative (NCSI; England) is improving management of the consequences of treatment, from diagnosis onwards. ${ }^{35}$ Likewise, survivorship, defined by the NCI, focuses on the health and life of a person with cancer post treatment until the end of life. It covers the physical, psychosocial, and economic issues of cancer; beyond the diagnosis and treatment phases. ${ }^{36}$ The combined late effects of myeloma and its treatment constitute a unique syndrome. Survivorship in myeloma therefore requires specialised screening, co-ordinated management and multi-disciplinary care. Holistic need assessment (HNA) will comprehensively assess physical, emotional, mental, spiritual and social concerns experienced by the individual and to formulate an individual care or action plan. ${ }^{37}$

As well as physical issues, psychological problems, social factors and concern about treatments and maintaining independence, all have a significant impact on HRQoL. ${ }^{38-40}$ Study showed that after 1 year follow-up, 75\% of myeloma patients have deteriorating HRQoL scores, with $37 \%$ worrying about their future health, $34 \%$ pre-occupied by their disease and $21 \%$ worrying about dying. ${ }^{41}$ Most HRQoL tools such as the European Organisation for Research and Treatment of Cancer Quality of Life (EORTC) QLQC30 and MY-20 questionnaires are used in clinical research rather than in routine clinical practice. ${ }^{42,43}$ The Myeloma Patient Outcome Scale (MyPOS) HRQoL questionnaire has been designed specifically for use in the clinical set-ting and focuses on the issues most important to patients. ${ }^{44}$ The importance of using structured HNA at key stages of cancer from diagnosis is now recognised. ${ }^{45}$

Myeloma patients are concerned by disease recurrence, loss of independence, and death. However, overall mental functioning remains preserved, suggesting effective coping mechanisms. ${ }^{46}$ In a survey of 114 patients, anxiety (8\%) and depression (24\%) were reported at the time of myeloma diagnosis and $51 \%$ of these patients had psychosocial intervention desires. The most common preferences were relaxation techniques, psychological counselling and peer support groups. ${ }^{47}$ Low mood, anxiety and clinical depression should be actively assessed and managed. A four-level model of psychological assessment and support is recommended by National Institute of Health and clinical Excellence (NICE) to guide escalation of professional intervention. ${ }^{48}$

Myeloma patients have the highest level of symptoms and the lowest level of HRQoL among patients with haematological cancers. ${ }^{49}$ The influence of fatigue and pain are the strongest predictors on HRQoL in myeloma ${ }^{50}$ and these long-term consequences of treatment are known to be the main barriers to social and participatory functions, even in stable phase disease. ${ }^{46,50,51}$ A European multi-centre study of myeloma patients reported that depression and fatigue had a negative effect on HRQoL; fatigue and bone pain were associated with poor physical functioning whilst mental status changes were associated with a reduction in social functioning score ${ }^{50}$ Exercise and physical activity may help; in a single arm pilot study, a tailored exercise programme was associated with increased muscle strength and improved fatigue scores. ${ }^{52}$ However, high quality trials are lacking, and existing evidence suggests that positive impacts on fatigue may be limited to the post-treatment period. . $^{53,54}$

Studies showed that only $20 \%$ of myeloma patients meet national physical activity guidelines post-treatment and $7 \%$ during active treatment. ${ }^{55,56}$ Commonly reported barriers to physical activity are pain, fear of fracture risk, lack of confidence and fatigue. ${ }^{52}$ Although more high quality randomised control trials (RCTs) are required in myeloma patients to determine efficacy ${ }^{53,54}$ exercise training has been shown to be safe and feasible with high attendance and adherence ${ }^{52,57}$ and higher physical activity levels have been associated with better quality of life both during and off active treatment. ${ }^{55,58}$ Rehabilitation in myeloma patients after HSCT improves physical performance, muscle strength, aerobic capacity and immunological function; reduces fatigue and improves psychological outcomes. ${ }^{52,59}$

Many times we do not formally assess myeloma patients for many trivial symptoms e.g. tiredness, sexual function etc. However one study showed that half of the patients reported tiredness as quite a bit/very much, while one third complained that daytime somnolence and insomnia were quite a bit/very much. Fortyfour percent of patients reported pain. One third of patients was bothered and distressed by the side effects from their treatment and was worried about long-term effects of their treatment. Thirty-one percent of patients felt that the effect of their condition had an impact on their sexual life, and $40 \%$ were worried about the effect that their illness was having on their family or other people. ${ }^{60}$ Studiesindicate the need for formal HNA for all myeloma patients. However, there is no properly designed prospective randomised trial specifically looking into the HNA for myeloma patients and I suggest it will be immensely beneficial to conduct such trial.

\section{Lymphoma and holistic needs assessment}

Lymphoma usually managed as a chronic disease as a result of high long-term survival rates. ${ }^{61}$ Survivors of lymphoma live with their disease and treatment effects for years; therefore, HRQoL is a central concern for clinical management. Compared to that of solid tumours, lymphoma survivorship has received little attention, but studies examining the course of morbidity in non-Hodgkin lymphoma (NHL) and Hodgkin lymphoma (HL) survivorship have revealed that these patients experience psychological disorders [e.g., anxiety, depression, post-traumatic stress disorder (PTSD)], ${ }^{62-64}$ delayed return to work ${ }^{65}$ and a subsequent decrease in their HRQoL. ${ }^{64,66}$ Beside these complications, other severe concerns include the development of cardiovascular diseases and second malignancies, while relapse also remains possible, especially during the first 24 months post-therapy. ${ }^{67}$

One study showed that the prevalence of anxiety was as high as $20.0 \%$ at three months but decreased over time $14.8 \%$ at twelve month. This study also showed that prevalence of depression was less frequent $9.6 \%$ at months three and $6.5 \%$ at month twelve. The prevalence of PTSD ranged between $14.8 \%$ of 115 patients at the beginning and $17.6 \%$ of at twelve month. Over the first 12 months, $42.6 \%$ of patients presented with at least one of the three psychological disorders anxiety, depression or PTSD. ${ }^{68}$

A recent study compared illness-related anxiety among participants in the Rituximab Extended Schedule or Retreatment Trial (RESORT) randomly assigned to maintenance rituximab (MR) versus rituximab re-treatment (RR). Illness-related anxiety was 
comparable between treatment arms at all time points regardless of coping style (active or avoidant). Illness-related anxiety and general anxiety significantly decreased over time on both arms. HRQoL scores were relatively stable and didnot change significantly from baseline for both arms. An avoidant coping style was associated with significantly higher anxiety $(18 \%$ and $13 \%$ exceeded clinical cut-off points at baseline and 6 months, respectively) and poorer HRQoL compared with an active coping style regardless of treatment arm assignment. ${ }^{69}$

\section{Discussion}

Applying a holistic approach to patient care has many benefits and yet, only around $25 \%$ of cancer survivors in the UK receive a holistic needs assessment and care plan. ${ }^{70,71}$ There is currently a concerted political, ethical and philosophical push towards improving patient experience and care in the UK NHS. ${ }^{72-74}$ and policy guidelines such as 'Improving supportive and palliative care for adults with cancer ${ }^{75}$ address the need to improve satisfaction, reduce distress, offer support and save money by facilitating selfcare. Improved collaboration between patient and clinician is central to this agenda. ${ }^{76}$ However, it is not clear how collaboration is optimised, who should be sharing what decisions, or how this may or may not impact on outcomes. ${ }^{77}$

Effective communication between the health professional and the patient is associated with improved psychological functioning of the patient, ${ }^{78,79}$ adherence to treatment and pain control ${ }^{80}$ and higher quality of life and satisfaction. ${ }^{81}$ By contrast, it has been suggested that poor communication may have a number of negative effects on the patient and the treatment process, including the nature and quality of information transmission, decision-making, and the psychosocial experience of the patient. ${ }^{82}$ Patient-reported outcome (PRO) measures are increasingly being used in routine cancer care. When a clinician reviews PRO measures with a patient during a visit, the patient has an opportunity to elaborate on symptoms that may need to be managed. Reviewing PRO measures with haematological cancer patients increases communication between clinicians and patients, detection of problematic symptoms, and satisfaction with care. ${ }^{83}$

In general haematological cancer survivors appear to report relatively good QoL in a range of conventional measures used in the general population. For instance, in a large study of adult survivors of childhood cancer, participants had similar QoL outcomes to those of the general population. ${ }^{84}$ However, most QoL measures comprise a generic list of physical symptoms, with or without some cancer site-specific symptoms and one or two items for psychological distress, and do not necessarily capture the experiences and subtle needs of long-term cancer survivors, including how current conditions in their lives may be attributed, related to or influenced by having had cancer. ${ }^{85}$ Recently, a QOL measure was developed to measure both positive and negative aspects of cancer survival and this scale has been used in US survivor groups ${ }^{86}$ as well as in Dutch patients. ${ }^{87}$

A large retrospective study of 718 long-term haematological cancer survivors assessed the impact of cancer (IOC) on subsequent quality of life (QoL) showed that fifteen percent survivors reported significant psychological distress, 18\% high levels of fatigue and $10 \%$ moderate to severe functional impairment. These groups of participants also showed poorer QoL. There were no significant differences in psychological distress across different cancer subtypes. Higher negative IOC scores were significantly associated with medical comorbidity, psychological distress, lower social support, high fatigue levels and functional impairment.
Paediatric patients (diagnosed at $<17$ years) had significantly higher negative IOC scores than adult patients; greater years since diagnosis was significantly associated with less negative IOC. Higher positive IOC was associated with acute leukaemia, lower positive IOC with paediatric patients, white ethnicity, higher education, no partner and lower social support. Screening for medical comorbidity, psychological distress and fatigue identifies those needing most support and should allow earlier interventions to address negative and positive IOC to improve the well-being of cancer survivors. ${ }^{6}$

Work productivity loss through haematological cancer has significant economic consequences for individuals, their families and society. ${ }^{88}$ More than this, the ability to work maintains financial stability, social relationships, self-esteem and psychological wellbeing. ${ }^{65,89-91}$ Two-thirds of patients with haematological malignancies return to work. However, the proportion is lowest for myeloma and acute leukaemia patients. Healthcare professionals can have a key influence on the likelihood of the patient's subsequent return to work ${ }^{92}$ and should take opportunities to assess the impact of disease and treatment consequences have on a person's ability to maintain their working life, and as a result, their identity and financial security. ${ }^{93}$

\section{Conclusions}

Patients with haematological cancer have a high prevalence of distress. Needs assessment should be performed routinely in clinical practice. Distress and unmet needs are common problems in these settings, and programs that routinely screen for and treat distress should be made available. The efforts of the last decade have established the importance of ensuring access to psychosocial services for haematological cancer survivors. Moving forward, we need to determine the most effective practices and how best to deliver them across diverse settings. Distress, like haematological cancer, is not a single entity, and one treatment does not fit all. Psychosocial-oncology needs to increase its research in comparative effectiveness. Health care providers need to be proactive to create successful systems in which all haematological cancer survivors will have some discussion with their providers about psychosocial care as well as physical health care, whether or not they choose to use psychosocial services.

\section{References}

1. Ferlay J, Soerjomataram I, Dikshit R, et al. Cancer incidence and mortality worldwide: sources, methods and major patterns in GLOBOCAN 2012. Int J Cancer 2014;136:E359-86.

2. Pulte D, Gondos A, Brenner H. Trends in survival after diagnosis with hematologicmalignancy in adolescence or young adulthood in the United States, 1981-2005. Cancer 2009;115:4973-9.

3. Maddams J, Utley M, Moller H. Projections of cancer prevalence in the United Kingdom, 2010-2040. Br J Cancer 2012; 107:1195-202.

4. Zabora J, Brintzenhofeszoc K, Curbow B, et al. The prevalence of psychological distress by cancer site. Psycho-oncol 2001;10:19-28.

5. Carlson L, Groff S, Maciejewski O, et al. Screening for distress in lung and breast cancer outpatients: A randomized controlled trial. J Clin Oncol 2010;28:4884-91. 
6. Korszun A, Sarker S, Chowdhury K, et al. Psychosocial factors associated with impact of cancer in long-term haematological cancer survivors. Br J Haematol 2014;164: 790-803.

7. Pirl WF, Muriel A, Hwang V, et al. Screening for psychosocial distress: a national survey of oncologists. J Support Oncol 2007;5:499-504.

8. Institute of Medicine. Cancer care for the whole patient: meeting psychosocial health needs. Washington, DC: National Academies Press; 2008.

9. Ferrell BR, Hassey Dow K. Measurement of the quality of life in cancer survivors. Qual Life Res 1995;4:523-31.

10. Baker F, Denniston M, Haffer SC, et al. Change in health-related quality of life of newly diagnosed cancer patients, cancer survivors, and controls. Cancer 2009;115:3024-33.

11. Baumann R, Pütz C, Röhrig B, et al. Health-related quality of life in elderly cancer patients, elderly non-cancer patients and an elderly general population. Eur J Cancer Care 2009; 18 : 457-65.

12. Kanellopoulos A, Hamre HM, Dahl AA, et al. Factors associated with poor quality of life in survivors of childhood acute lymphoblastic leukemia and lymphoma. Pediatric Blood Cancer 2013;60:849-55.

13. Timperi AW, Ergas IJ, Rehkopf DH, et al. Employment status and quality of life in recently diagnosed breast cancer survivors. Psycho-oncol 2013;22:1411-20.

14. Jarrett N, Scott I, Addington-Hall J, et al. Informing future research priorities into the psychological and social problems faced by cancer survivors: a rapid review and synthesis of the literature. Eur J Oncol Nurs. 2013;17:510-20.

15. Montazeri A. Quality of life data as prognostic indicators of survival in cancer patients: an overview of the literature from 1982 to 2008. Health Qual Life Outcomes 2009;7:102.

16. Redaelli A, Stephens JM, Brandt S, et al. Short- and long-term effects of acute myeloid leukemia on patient health-related quality of life. Cancer Treat Rev 2004;30:103-17.

17. Efficace F, Kemmler G, Vignetti M, et al. Health-related quality of life assessment and reported outcomes in leukaemia randomised controlled trials - a systematic review to evaluate the added value in supporting clinical decision making. Eur $\mathrm{J}$ Cancer 2008;44:1497-506.

18. Battaglini CL, Hackney AC, Garcia R, et al. The effects of an exercise program in leukemia patients. Integr Cancer Ther 2009;8:130-8.

19. Oliva EN, Nobile F, Alimena G, et al. Quality of life in elderly patients with acute myeloidleukemia: patients may be more accurate than physicians. Haematologica 2011;96:696-702.

20. Bieri S, Roosnek E, Helg C, et al. Quality of life and social integration after allogeneic hematopoietic SCT. Bone Marrow Transpl 2008;42:819-27.

21. Watson M, Buck G, Wheatley K, et al. Adverse impact of bone marrow transplantation on quality of life in acute myeloid leukaemia patients; analysis of the UK Medical Research Council AML 10 Trial. Eur J Cancer 2004;40:971-8.

22. Moller T, Adamsen L, Appel C, et al. Health related quality of life and impact of infectious comorbidity in outpatient management of patients with acute leukemia. Leuk Lymph 2012;53:1896-904.

23. Leunis A, Redekop WK, Uyl-de Groot CA, et al. Impaired health-related quality of life in acute myeloid leukemia survivors: a single-center study. Eur J Haematol 2014;93:198206.

24. Schultz KAP, Chen L, Chen Z, et al. Health conditions and quality of life in survivors of childhood acute myeloid leukemia comparing post remission chemotherapy to BMT: a report from the children's oncology group. Pediatr Blood Cancer 2014;61:729-36.

25. Sekeres MA, Stone RM, Zahrieh D, et al. Decision-making and quality of life in older adults with acute myeloid leukemia or advanced myelodysplastic syndrome. Leukemia 2004;18:809-16.

26. Gupta V, Panzarella T, Li L, et al. A prospective study comparing the outcomes and health-related quality of life in adult patients with myeloid malignancies undergoing allogeneic transplantation using myeloablative or reduced-intensity conditioning. Biol Blood Marrow Transplant 2012;18:113-24.

27. Kurosawa S, Yamaguchi T, Mori T, et al. Patient-reported quality of life after allogeneic hematopoietic cell transplantation or chemotherapy for acute leukemia. Bone Marrow Transplant 2015;50:1241-9.

28. Parkin M, Bray F, Ferlay J, Pisani P. Global cancer statistics, 2002. CA Cancer J Clin 2005;55:74-108.

29. Bird J, Owen R, D'Sa S, et al. Guidelines for the diagnosis and management of multiple myeloma 2011. Br J Haematol 2011;154:32-75.

30. Gulbrandsen N, Wisloff F, Brinch L, et al. Health-related quality of life in multiple myeloma patients receiving high-dose chemotherapy with autologous blood stem-cell support. Med Oncol 2001;18:65-77.

31. Sherman AC. Psychosocial adjustment and quality of life among multiple myeloma patients undergoing evaluation for autologous stem cell transplantation. Bone Marrow Transplant 2004;33:955-62.

32. Uyl-de Groot CA, Buijt I, Gloudemans IJM, et al. Health related quality of life in patients with multiple myeloma undergoing a double transplantation. Eur J Haematol 2005;74:136-43.

33. Sherman AC, Simonton S, Latif U, et al. Changes in quality of life and psychosocial adjustment among multiple myeloma patients treated with high-dose melphalan and autologous stem cell transplantation. Biol Blood Marrow Transplant 2009;15:12-20.

34. Osborne TR, Ramsenthaler C, de Wolf-Linder S, et al. What matters most to people with multiple myeloma? A qualitative study of views on quality of life. BMC Cancer 2014;14:496.

35. Richards M, Corner J, Maher J. The National Cancer Survivorship Initiative: new and emerging evidence on the ongoing needs of cancer survivors. Br J Cancer 2011;105:S14.

36. National Cancer Institute. NCI Dictionary of Cancer Terms. Available from: http://www.cancer.gov/dictionary

37. Richardson A, Medina J, Brown V, et al. Patients' needs assessment in cancer care: a review of assessment tools. Supportive Care in Cancer 2007; 15:1125-44.

38. Frodin U, Borjeson S, Lyth J, et al. A prospective evaluation of patients' health-related quality of life during auto-SCT: a 3year follow-up. Bone Marrow Transplant 2010;46:1345-52.

39. Molassiotis A, Wilson B, Blair S, et al. Living with multiple myeloma: experiences of patients and their informal caregivers. Support Care Cancer 2011;19:101-11.

40. Molassiotis A, Wilson B, Blair S, et al. Unmet supportive care needs, psychological well-being and quality of life inpatients living with multiple myeloma and their partners. Psychooncol 2011;20:88-97.

41. Mols F, Oerlemans S, Vos A, et al. Health-related quality of life and disease-specific complaints among multiple myeloma patients upto $10 \mathrm{yr}$ after diagnosis: results from a populationbased study using the PROFILES registry. Eur J Haematol 2012;89:311-9.

42. Kvam A, Fayers P, Hjermstad M, et al. Health-related quality 
of life assessment in randomised controlled trials in multiple myeloma: a critical review of methodology and impact on treatment recommendations. Eur J Haematol 2009;83:279-89.

43. Osborne T, Ramsenthaler C, Siegert R, et al. What issues matter most to people with multiple myeloma and how well are we measuring them? A systematic review of quality of life tools. Eur J Haematol 2012;89:437-57.

44. Osborne $\mathrm{T}$, Ramsenthaler $\mathrm{C}$, Ramsenthaler $\mathrm{C}$, et al. Improving the assessment of quality of life in the clinical care of myeloma patients: the development and validation of the Myeloma Patient Outcome Scale (MyPOS). BMC Cancer 2015;15:280.

45. Department of Health, Macmillan Cancer Support \& NHS Improvement. Living with and Beyond Cancer: Taking Action to Improve Out-comes (an update to the 2010 The National Cancer Survivorship Initiative Vision). Gateway reference 18455. London: Department of Health; 2013.

46. Boland E, Eiser C, Ezaydi Y, et al. Living with advanced but stable multiple myeloma: a study of the symptom burden and cumulative effects of disease and intensive (hematopoietic stem cell transplant-based) treatment on health-related quality of life. J Pain Sympt Manage 2013;46:671-80.

47. Lamers J, Hartmann M, Goldschmidt H, et al. Psychosocial support in patients with multiple myeloma at time of diagnosis: who wants what? Psychooncology 2013;22:2313-20.

48. NICE. Improving supportive and palliative care for adults with cancer. Guidance on Cancer Services. London: National Institute for Clinical Excellence; 2004. Available from: https://www.nice.org.uk/guidance/csg4/resources/improvingsupportive-and-palliative-care-for-adults-with-cancer773375005

49. Johnsen A, Tholstrup D, Petersen MA, et al. Health related quality of life in a nationally representative sample of haematological patients. Eur J Haematol 2009;83:139-48.

50. Jordan K, Proskorovsky I, Lewis P, et al. Effect of general symptom level, specific adverse events, treatment patterns, and patient characteristics on health-related quality of life in patients with multiple myeloma: results of a European, multicenter cohort study. Support Care Cancer 2014;22:417-26.

51. Gulbrandsen N, Hjermstad M, Wisløff F. Nordic Myeloma Study Group. Interpretation of quality of life scores in multiple myeloma by comparison with a reference population and assessment of the clinical importance of score differences. Eur J Haematol 2004;72:172-80.

52. Groeneveldt L, Mein G, Garrod R, et al. A mixed exercise training programme is feasible and safe and may improve quality of life and muscle strength in multiple myeloma survivors. BMC Cancer 2013;13:31.

53. Smith L, McCourt O, Henrich M, et al. Multiple myeloma and physical activity: a scoping review. BMJ Open 2015;5:e009576.

54. Gan J, Sim C, Santorelli L. The effectiveness of exercise programmes in patients with multiple myeloma: a literature review. Crit Rev Oncol Hematol 2015;98:275-89.

55. Jones L, Courneya K, Vallance J, et al. Association between exercise and quality of life in multiple myeloma cancer survivors. Support Care Cancer 2004;12:780-8.

56. Craike M, Hose K, Courneya K, et al. Perceived benefits and barriers to exercise for recently treated patients with multiple myeloma: a qualitative study. BMC Cancer 2013;13:319.

57. Shallwani S, Dalzell M, Sateren W, et al. Exercise compliance among patients with multiple myeloma undergoing chemotherapy: a retrospective study. Support Care Cancer 2015;23:30818.

58. Groeneveld I, de Boer A, Frings-Dresen M. Physical exercise and return to work: cancer survivors' experiences. J Cancer Survivor Res Pract 2013;7:237-46.

59. Persoon S, Kersten M, van der Weiden K, et al. Effects of exercise in patients treated with stem cell transplantation for a hematologic malignancy: a systematic review and meta-analysis. Cancer Treat Rev 2013;39:682-90.

60. Boland E, Boland J, Ezaydi Y, et al. Holistic needs assessment in advanced, intensively treated multiple myeloma patients. Support Care Cancer 2014;22:2615-20.

61. Centers for disease control and prevention. Cancer survivorship - United States, 1971-2001. MMWR 2004;53:526-9.

62. Smith SK, Mayer DK, Zimmerman S, et al. Quality of life among long-term survivors of non-Hodgkin lymphoma: a follow-up study. J Clin Oncol 2013;31:272-9.

63. Smith SK, Zimmerman S, Williams CS, et al. Post-traumatic stress symptoms in long-term non-Hodgkin'slymphoma survivors: does time heal? J Clin Oncol 2011;29:4526-33.

64. Oerlemans S, Mols F, Nijziel MR, et al. The course of anxiety and depression for patients with Hodgkin's lymphoma or diffuse large B cell lymphoma: a longitudinal study of the PROFILES registry. J Cancer Surviv Res Pract 2014;8:555-64.

65. Horsboel TA, Nielsen CV, Nielsen B, et al. Type of hematological malignancy is crucial for the return to work prognosis: a register-based cohort study. J Cancer Surviv Res Pract 2013; 7:614-23.

66. Jensen RE, Arora NK, Bellizzi KM, et al. Health-related quality of life among survivors of aggressive non-Hodgkin lymphoma. Cancer 2013;119:672-80.

67. Maurer MJ, Ghesquières H, Jais J-P, et al. Event-free survival at 24 months is a robust end point for disease-related outcome in diffuse large B-cell lymphoma treated with immunechemotherapy. J Clin Oncol 2014;32:1066-73.

68. Compaci G, Rueter M, Lamy S, et al. Ambulatory Medical Assistance - After Cancer (AMA-AC): A model for an early trajectory survivorship survey of lymphoma patients treated with anthracycline-based chemotherapy. BMC Cancer 2015; 15:781.

69. Wagner L, Zhao F, Hong F, et al. Anxiety and Health-Related Quality of Life Among Patients With Low Tumor Burden NonHodgkin Lymphoma Randomly Assigned to Two Different Rituximab Dosing Regimens: Results From ECOG Trial E4402 (RESORT). J Clin Oncol 2015;33:740-8.

70. Snowden A, White C. Assessment and care planning for cancer survivors: a concise evidence review. Macmillan Cancer Support, 2014. Available from: http://be.macmillan.org.uk/be/p-21255-assessment-and-careplanning-for-cancer-survivors-a-concise-evidence-review.aspx

71. Brennan J, Gingell P, Brant H, et al. Refinement of the distress management problem list as the basis for a holistic therapeutic conversation among UK patients with cancer. Psychooncology 2012;21:1346-56.

72. Coulter A, Collins A. Making shared decision making a reality. No decision about me, without me. London: The King's Fund; 2011.

73. The Scottish Government. The Quality Strategy; 2010. Available from: http:/www.scotland.gov.uk/Topics/Health/ NHS-Scotland/NHSQuality/Quality Strategy

74. Scottish Executive. Better cancer care: an action plan. Edinburgh: Scottish Government; 2008.

75. National Institute for Health and Clinical Excellence. Improving supportive and palliative care for adults with cancer; 2004. Available from: http://www.nice.org.uk/ guidance/csgsp

76. Elwyn G, Barr PJ, Grande SW, et al. Developing 
CollaboRATE: a fast and frugal patient-reported measure of shared decision making in clinical encounters. Patient Educ Couns 2013;93:102-7.

77. Cribb A. Involvement, shared decision-making and medicines. London: Royal Pharmaceutical Society; 2011.

78. McCormack LA, Treiman K, Rupert D, et al. Measuring patient-centered communication in cancer care: a literature review and the development of a systematic approach. Soc Sci Med 2011;72:1085-95.

79. Ford S, Fallowfield L, Lewis S. Doctor-patient interactions in oncology. Soc Sci Med 1996;42:1511-9.

80. Brown JE, Brown RF, Miller RM, et al. Testing health care professionals' communication skills: the usefulness of highly emotional standardized role-playing sessions with simulators. Psychooncology 2000;9:293-302.

81. Fukui S, Ogawa K, Yamagishi A. Effectiveness of communication skills training of nurses on the quality of life and satisfaction with healthcare professionals among newly diagnosed cancer patients: a preliminary study. Psychooncology 2011;20:1285-91.

82. Thorne S, Bultz BD, Baile WF. Is there a cost to poor communication in cancer care?: a review of the literature. Psychooncology 2005; 14:875-84.

83. Kotronoulas G, Kearney N, Maguire R, et al. What is the value of the routine use of patient-reportedoutcome measures toward improvement of patient outcomes, processes of care, and health service outcomes in cancer care? A systematic review of controlled trials. J Clin Oncol 2014;32:1480-501.

84. Reulen R, Frobisher C, Winter DL, et al. British Childhood Cancer Survivor Study Steering Group Long-term risks of subsequent primary neoplasms among survivors of childhood cancer. JAMA 2011;305:2311-19.

85. Oerlemans S, Mols F, Nijziel MR, et al. The impact of treatment, socio-demographic and clinical characteristics on health-related quality of life among Hodgkin's and nonHodgkin's lymphoma survivors: a systematic review. Ann Hematol 2011;90:993-1004.

86. Crespi CM, Smith SK, Petersen L, et al. Measuring the impact of cancer: a comparison of non-Hodgkin lymphoma and breast cancer survivors. J Cancer Survivorship 2010;4:45-58.

87. Oerlemans S, Smith SK, Crespi CM, et al. Assessing the impact of cancer among Dutch non-Hodgkin lymphoma survivors compared with their American counterparts: a crossnational study. Psychooncology 2013;22:1258-65.

88. Luengo-Fernandez R, Leal J, Gray A, et al. Economic burden of cancer across theEuropean Union: a population-based cost analysis. Lancet Oncol 2013;14:1165-74.

89. Goodwin J, Coleman E, Sullivan E, et al. Personal financial effects of multiple myelomaand its treatment. Cancer Nursing 2013;36:301-8.

90. Timmons A, Gooberman-Hill R, Sharp L, et al. The multidimensional nature of thefinancial and economic burden of a cancer diagnosis on patients and their families: qualitativefindings from a country with a mixed public-private healthcare system. Support Care Cancer 2013;21:107-17.

91. Wells M, Williams B, Firnigl D, et al. Supporting 'work-related goals' rather than 're-turn to work' after cancer? A systematicreviewand meta-synthesis of 25 qualitative studies. Psycho-oncology 2013;22:1208-19.

92. Pryce J, Munir F, Haslam C. Cancersurvivorship and work: symptoms, supervisorresponse, co-worker disclosure and work adjustment. J Occupat Rehab 2007;17:83-92.

93. Wells M, Amir Z, Cox T, et al. Timeto act: the challenges of working during andafter cancer, initiatives in research and practice. Guest Editorial. Eur J Oncol Nursing 2014;18:1-2. 